Laurent Faure

École nationale supérieure de formation de

l'enseignement agricole de Toulouse

France

Cécile Gardiès

École nationale supérieure de formation de

l'enseignement agricole de Toulouse

France

Jean-François Marcel

Université Toulouse - Jean-Jaurès

France

\section{Formation des enseignants : apprentissages professionnels d'enseignants novices au travers de régulations en situation de classe}

doi:10.18162/fp.2017.403

\section{ésumé}

La question de la formation des enseignants novices en situation se pose de manière de plus en plus cruciale au vu des réformes récentes qui instaurent une entrée rapide dans le métier. Nous interrogeons ici la construction de savoirs professionnels. Un dispositif de régulation en situation entre enseignant expérimenté et enseignant novice permet d'appréhender la transformation de la connaissance en relation avec le sentiment d'efficacité professionnelle. Les résultats montrent que, dans les différentes catégories de régulation, on peut mettre en avant une transformation de la connaissance dotée d'une imprévisibilité irréductible, d'un développement dans l'action, pour celle-ci et qui prend en charge la question de la responsabilité du sujet connaissant.

Mots-clés

Enseignants novices, intention, modification de l'action, pratiques d'enseignement, réflexivité sur les pratiques, régulation en situation d'enseignement

Abstract

The on-site training of aspiring professors is becoming a key issue as the recent legal evolutions push toward quicker entries into the profession. We wonder in this article about the construction of professional knowledge in class regulation system between an aspiring professor and an experimented professor enables a knowledge transformation together with a rewarding professional efficiency feeling. Results show that across different types of regulation a knowledge transformation occurs, though coupled with unavoidable unpredictability. A clear growth in and for the action is also seen, that addresses the question of the responsibility of the knowing subject.

Keywords

Aspiring professors, willingness, action alteration, teaching methods, reflexivity about practices, in class teaching

regulation

\section{Introduction}

Dans le contexte actuel de changement dans la formation des enseignants, il nous paraît important, à la suite d'autres travaux menés sur cette même thématique (Bru, 2004; Marcel, 2004), d'interroger les pratiques des enseignants novices et la manière dont les savoirs professionnels sont construits et partagés dans le cadre de l'entrée dans le métier. En effet, les enseignants sont amenés à exercer leur fonction très vite après l'obtention d'un niveau disciplinaire qui les certifie sans avoir un temps de formation et d'accompagnement pédagogique et didactique conséquent. Cette situation se retrouve également dans le cas des enseignants ayant un master $\mathrm{MEEF}^{1}$ tel qu'il a été mis en place à la rentrée 2015 dans l'enseignement agricole français.

Certaines disciplines scolaires, de par leurs spécificités, accentuent ces difficultés. C'est le cas par exemple de la discipline sciences et techniques des agroéquipements $\left(\mathrm{STAE}^{2}\right.$ ) (présente dans les programmes scolaires de l'enseignement agricole en France), où les situations d'enseignement-apprentissage sont encore plus diversifiées, car liées d'une part à des espaces d'enseignement variés et, d'autre part, à des savoirs et à des pratiques aux références scientifiques et techniques multiples. En effet, les savoirs à mobiliser pour enseigner en STAE restent peu stabilisés au regard de leur complexité, de leur multi-référentialité (Gillet et Fauré, 2014), et des contraintes et injonctions liées à ces enseignements particuliers. Il est donc difficile pour les enseignants débutants dans cette discipline de construire les savoirs professionnels nécessaires dans le contexte d'une mise en situation ou d'une entrée rapide dans le métier. Par ailleurs, les conseillers pédagogiques ${ }^{3}$ qui les accompagnent ont assez peu de temps depuis la réforme de la masterisation ${ }^{4}$ pour former les enseignants en situation, ce qui complexifie la fonction d'étayage. 
Pour étudier la construction de savoirs professionnels des enseignants novices au travers de régulation en situation de classe, nous proposons de présenter le cadre théorique des pratiques d'enseignement en mettant en avant le sentiment d'efficacité professionnelle, les savoirs professionnels qui s'y rattachent et la conversion de la connaissance dans l'action. D'un point de vue méthodologique, dans une approche qualitative, nous appuyons notre collecte d'éléments empiriques sur un dispositif de régulation en situation d'enseignement (qui pourrait se référer aux dispositifs existants de «bug-in-ear ", Rock et al., 2009). Ce dispositif nous permet de réaliser des observations de séances régulées et des entretiens auprès des enseignants. La collecte porte sur 6 séances et sur 3 enseignants. Pour analyser ces éléments empiriques à partir du principe de la double lecture des pratiques (Lefeuvre, 2005), nous mettrons en relation les régulations observées avec le sentiment d'efficacité professionnelle préalablement déclaré et les discours sur ces régulations recueillis postérieurement aux séances régulées. Nous discuterons ces résultats au regard du cadre d'analyse de la conversion de la connaissance comme potentiellement constitutive de la co-construction de savoirs professionnels dans le processus de formation des enseignants.

\section{Problématisation}

Toute situation d'enseignement-apprentissage est complexe. En effet, les enseignants doivent prendre en compte un ensemble de facteurs et percevoir de nombreux indices pour adapter leurs pratiques (Wanlin et Crahay, 2012). Ce constat est particulièrement repérable chez l'enseignant expérimenté (que nous nommerons «enseignant $\mathrm{E}$ » dans cet article) qui utilise son expérience pour saisir et sélectionner un nombre d'indices relativement limité qui lui permet de s'adapter au fur et à mesure de la situation (Tochon, 2004). Pour prendre en compte cette complexité et pouvoir agir en conséquence, les enseignants procèdent en fonction de facteurs liés aux élèves, de facteurs liés aux savoirs et aux gestes de métier qui peuvent s'appréhender au travers de leur sentiment d'efficacité professionnelle (Marcel, 2009). C'est dans ce contexte que se pose la question de la construction de savoirs professionnels chez les enseignants novices (que nous nommerons « enseignants $\mathrm{N}$ » dans cet article) qui leur permet d'aborder les situations d'enseignement complexes. Le terme " savoirs professionnels » est polysémique et renvoie à une grande variété de sens. On peut cependant retenir avec Tardif et Lessard (1999) que les savoirs professionnels se définissent comme l'ensemble des connaissances, compétences, aptitudes et attitudes à la base de l'enseignement. Or, les enseignants $\mathrm{N}$ peuvent avoir des difficultés pour percevoir et sélectionner les indices dans la situation d'enseignement pour agir, en particulier parce qu'ils n'ont pas encore acquis tous les savoirs nécessaires. De plus, les enseignants E ne savent pas toujours montrer aux enseignants $\mathrm{N}$ les étapes qui leur ont permis d'acquérir ces savoirs et construire ainsi une expertise qui est notamment basée sur la sélection d'indices pertinents (Fauré, 2014).

Le processus progressif de construction des savoirs professionnels, particulièrement chez les enseignants $\mathrm{N}$, s'opère à la fois en situation d'enseignement-apprentissage et en situation de formation. Mais ce qui caractérise les situations d'enseignement complexes est leur taux de variabilité important corrélé à la diversité des contextes, ce qui limite leur prévisibilité et demande une adaptation et une forte mobilisation de savoirs professionnels. Ce type de savoirs semble s'acquérir plus par l'expérience et sa conceptualisation qu'en amont en formation initiale. Or, la confrontation à des situations réelles d'enseignement est frontale et ne peut donc guère se préparer en situation de laboratoire. De plus, 
cette confrontation se fait majoritairement de manière « solitaire », ce qui ne favorise pas la réflexion distanciée sur sa propre expérience.

Si l'on considère comme nécessaire la construction d'un certain nombre de savoirs professionnels au-delà de l'expérience, comment alors accompagner les enseignants $\mathrm{N}$ directement en situation? Si mettre en place des dispositifs d'accompagnement peut constituer une manière de participer à la construction de savoirs professionnels, pour autant comment circulent ces savoirs? À partir de quels types de régulations? En quoi ces régulations en situation de classe permettent-elles de convertir la connaissance? Quels sont les savoirs professionnels qui se développent à partir de cette conversion?

\section{Cadre théorique}

\section{a) Pratiques d'enseignement}

Nous nous intéressons ici aux pratiques d'enseignement, comme un sous-ensemble des pratiques enseignantes, et aux pratiques de l'enseignant face aux élèves (Marcel, 2002).

Les pratiques d'enseignement sont ainsi inscrites dans une filiation de la théorie sociale et cognitive développée par Bandura (1977). Dans cette approche, «le fonctionnement humain est le produit d'une interaction dynamique et permanente entre des cognitions, des comportements et des circonstances environnementales» (Carré, 2004). Il présente ainsi le modèle de la "causalité triadique réciproque $»$ :

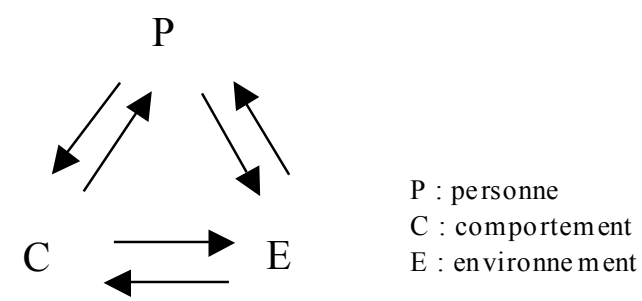

Modèle de causalité triadique réciproque (Bandura, 1986).

Dans celui-ci, les interactions entre le comportement et les facteurs personnels, le comportement et l'environnement ainsi que les facteurs internes sont de nature réciproque et s'influencent mutuellement (Bandura, 1986).

Dans le prolongement de ce modèle, Bandura (1986) précise que pour s'adapter à des situations complexes et variées, la croyance en son efficacité ainsi que l'évaluation de ses capacités sont des éléments clés favorisant l'appréhension de la situation par un individu. Le sentiment d'efficacité personnelle est un des éléments qui organise l'action d'un individu dans un environnement donné. Dans le domaine des pratiques d'enseignement, nous nous appuyons sur la proposition de sentiment d'efficacité non plus personnelle, mais professionnelle (Marcel, 2009), non pas comme un indicateur de développement professionnel (Marcel, 2009) de l'enseignant, ni comme un organisateur des pratiques d'enseignement (Safourcade, 2010), mais pour étudier les liens entre les savoirs préalablement acquis ou pensant l'être 
et les contenus des régulations des pratiques d'enseignement en situation de classe potentiellement vecteurs de nouvelles connaissances.

En complément et en suivant en cela la proposition de Marcel (2014), nous considérons les pratiques enseignantes comme un ensemble dynamique de processus. Les quatre composantes du modèle, qui " génèrent » (sont à l'origine de) ces processus, sont par conséquent repérés comme des " processeurs » (dans un sens commun, assez proche de celui de l'informatique) et définis par :

- $\quad$ le processeur ontologique (le qualificatif « ontologique » correspond ici au « sujet dans le prolongement de la "spirale de la connaissance" » (Nonaka et Von Krogh, 2009). Elle a été mobilisée pour l'élaboration du modèle des pratiques et a permis de dépasser la théorie de l'agentivité développée par Bandura).

- le processeur environnemental, avec ces différents niveaux : spatiaux, temporels, matériels, sociaux, organisationnels, institutionnels, culturels, historiques, symboliques, etc.

- $\quad$ le processeur praxique, celui des gestes, des comportements et des discours en situation;

- le processeur épistémologique, celui des connaissances et des savoirs, ceux de l'enseignant, mais aussi celui des savoirs mis en jeu dans les situations d'enseignement.

Le processeur épistémologique nécessite de caractériser les savoirs en circulation pour mieux comprendre les enjeux des régulations pour les enseignants $\mathrm{N}$ et plus généralement pour la formation des enseignants.

\section{b) Les savoirs des enseignants}

Nous utiliserons le terme de "savoirs professionnels » comme un terme générique qui désigne les savoirs issus d'un enseignant, comme les savoirs construits par les enseignants au fur et à mesure de leurs expériences vécues.

Gauthier, Desbiens, Malo, Martineau et Simard (1997) identifient six types de savoirs des enseignants : les savoirs disciplinaires, curriculaires, des sciences de l'éducation, de la tradition pédagogique, d'expérience et d'action pédagogique. Pour eux, le savoir disciplinaire fait référence aux savoirs produits par les chercheurs et les savants dans les différentes disciplines scientifiques. Le savoir curriculaire concerne la connaissance du programme d'enseignement, c'est-à-dire le savoir disciplinaire transformé par la structure, l'organisation et les manuels scolaires. Le savoir des sciences de l'éducation renvoie à certaines connaissances professionnelles ne concernant pas directement l'action pédagogique, comme la connaissance du système scolaire, du syndicat, du régime pédagogique. Le savoir de la tradition pédagogique, comme son nom l'indique, est un savoir issu de la coutume. Le savoir d'expérience fait référence à l'expérience ou plutôt aux expériences vécues par chaque enseignant dans l'exercice de sa profession. Ces expériences deviennent en quelque sorte une jurisprudence « privée » pour l'enseignant. Cette jurisprudence devient savoir d'action pédagogique quand elle est rendue publique et cautionnée par la recherche qui se fait en classe. Ce savoir d'action pédagogique consiste essentiellement, selon les auteurs, en la gestion de la matière et la gestion de la classe. 
Pour Tardif et Lessard, il est souhaitable de proposer un modèle construit à partir des catégories des praticiens eux-mêmes et des connaissances qu'ils utilisent dans leur pratique professionnelle : « enseigner, c'est mobiliser une large variété de connaissances composites, en les réinvestissant dans le travail, pour les adapter et les transformer pour et par le travail (Tardif et Lessard, 1999). Les savoirs professionnels peuvent donc être considérés comme « des savoirs pertinents dans un métier ou une profession, quelle qu'en soit la source, qu'ils viennent de la recherche, de la culture professionnelle ou de l'expérience personnelle» (Perrenoud, 2000).

Cet éclairage sur la notion de savoirs professionnels, à la fois au service et incorporé dans la pratique interroge fortement la manière dont ils peuvent être acquis, construits et partagés. Nous proposons de nous référer à la théorie de la conversion de la connaissance principalement développée par Nonaka (1994), pour envisager d'abord de manière théorique comment les savoirs se transforment dans l'action. Précisons en amont que les termes de savoir et de connaissance ne font pas référence aux mêmes sens. Ainsi en France, une différenciation est faite entre information, connaissance et savoir (Astolfi, 1995; Gardiès, 2012; Jeanneret, 2000), même si dans le monde anglo-saxon un seul terme regroupe connaissances et savoir, soit celui de «knowledge ». Nous avons cependant noté une différenciation faite entre information, explicit knowledge, tacit knowledge (Nonaka et Von Krogh, 2009) qui peut rejoindre la différenciation des termes en français. La connaissance est propre à l'individu, elle se construit à partir de l'information et se transmet par l'information. Le savoir est la somme des connaissances socialement reconnues, il constitue un tout objectivé. La connaissance revêt un caractère personnel et subjectif, alors que, comme le définit Foucault, le savoir est « cet ensemble d'éléments, formés de manière régulière par une pratique discursive et qui sont indispensables à la constitution d'une science »(Foucault, 1975).

\section{c) Transformation des savoirs}

Les modèles de partage et de circulation des connaissances au sein d'organisations développés notamment par Nonaka et Peltokorpi (2006) ainsi que Nonaka et Von Krogh (2009) permettent d'analyser comment se construit la connaissance organisationnelle, comment elle se transforme et en quoi elle participe à la performance individuelle, mais aussi collective. La connaissance organisationnelle peut se définir comme une somme de connaissances mises en commun sur lesquelles les acteurs pourront avoir une approche signifiante (prenant sens dans son application), c'est-à-dire inscrite dans une dynamique d'utilisation, de transmission et d'appropriation. La création ou la transformation de la connaissance organisationnelle, en s'appuyant sur cette mise en commun des connaissances individuelles, suppose de comprendre quelle est la nature des connaissances en jeu de manière à approcher ces processus. Ces modèles nous paraissent également opérants dans le cadre d'une organisation dédiée à la formation d'enseignants (Gardiès et Marcel, 2013) d'autant que le partage des connaissances est au centre de sa dynamique. Contrairement à d'autres modèles, ces théories ont développé des modèles de conversion ou de création de la connaissance basés sur un processus dynamique (en spirale) entre connaissances tacites et connaissances explicites sur des dimensions à la fois épistémologique et ontologique (Nonaka, 1994). Ces processus se déroulent en général à partir de trois axes. Le premier axe concerne la dimension épistémologique, c'est-à-dire la nature des connaissances en circulation et leur appropriation par les acteurs. Le deuxième axe est la dimension ontologique, soit la dimension sociale de partage et de 
construction de connaissances, la dimension de la création et de la transformation de la connaissance organisationnelle par le sujet. Enfin, le troisième axe est la dimension praxéologique référant à l'action des individus autour de la connaissance partagée (Gardiès et Marcel, 2013).

Si la connaissance tacite relève des capacités, de l'intuition de règles et de savoir-faire implicites, subjectifs, elle diffère de la connaissance explicite qui a un caractère universel, objectivé.

The concept of tacit knowledge is a corner store in organizational knowledge creation theory and covers knowledge that is unarticuled and tied to the senses, movement skills, physical experiences, intuition, or implicit rules of thumb [...] Tacit knowledge differs from "explicit knowledge" that is uttered and captured in drawings and writing is explicit. Explicit knowledge has a universel character. (Nonaka et Von Krogh, 2009).

L'explicitation d'une connaissance personnelle pour la partager devient une information transmise qui permet la diffusion de cette connaissance et l'appropriation par autrui, donc la constitution d'une nouvelle connaissance :

Knowledge tied to thesenses, tactile experiences, movementskills, intuition, unarticuled mental models, or implicit rules of thumb is "tacit". Tacit knowledge is rooted in action, procedures, routines, commitments, ideals, values, and emotions. Tacit knowledge can be accessible through consciousness if it leans towards the explicit side of continuum [...] the notion of continuum refers to knowledge ranging from tacit to explicit and vice versa. By incorporating "tacit knowledge" organizational knowledge creation theory overcame mainstream theory's tendency to equate knowledge with information (Nonaka et Von Krogh, 2009).

La connaissance tacite peut être partagée et ainsi transformée en connaissance explicite.

Les processus de création de la connaissance organisationnelle ne sont jamais « finis " : il s'agit d'un processus circulaire qui d'ailleurs n'est pas limité à l'organisation, mais inclut beaucoup d'interfaces avec l'environnement (Nonaka, 1994). Ce modèle, même s'il paraît toujours pertinent pour approcher le processus de création ou de transformation de la connaissance organisationnelle et par là même comprendre la construction des connaissances au sein d'une organisation, s'est enrichi au fur et à mesure de l'avancée des recherches d'autres éléments. Nous en retiendrons deux, celui de la prise en compte des pratiques sociales, car « organizational knowledge creation is very sensitive to social context » (Nonaka et Von Krogh, 2009) et celui du continuum objectivité-subjectivité entre connaissances tacites et connaissances explicites même si « social practices may be necessary, but not sufficient, for understanding organizational knowledge creation » (Nonaka et Von Krogh, 2009).

La connaissance, en circulant, engage des processus de signification socialement construits, c'està-dire basés sur les interactions sociales constructives. Le processus de création de la connaissance organisationnelle est un processus dans lequel la connaissance individuelle est partagée, amplifiée puis réinternalisée en tant que partie de la connaissance organisationnelle de base (Nonaka et Peltokorpi, 2006). Autrement dit, nous pouvons considérer que dans les processus de partage et de circulation des connaissances au sein des organisations de formation, il y a une dimension épistémologique relative à la nature des connaissances, la dimension ontologique relative au sujet connaissant et la dimension praxéologique relative aux pratiques sociales.

77 - Formation et profession 25(3), 2017 
En synthèse, considérons avec Gardiès et Marcel (2013) que nous pouvons déterminer trois axes pour la transformation des connaissances :

\# Une connaissance (axe épistémologique) incarnée et mobilisée (plan onto-praxéologique) qui dote le processus de conversion d'une touche d'imprévisibilité irréductible (celle de l'action en contexte) et qui le rapproche des problématiques du changement,

\# Une action (axe praxéologique) incarnée et éclairée (plan onto-épistémologique) qui émancipe le processus de conversion de la réflexivité (chère à Schön et Argyris) et qui souligne, à l'instar des néopiagétiens, le développement de la connaissance dans et par l'action,

\# Un sujet (axe ontologique) connaissant et agissant (axe épistémo-praxéologique) qui contraint le processus de conversion à prendre en charge la question de la responsabilité du sujet connaissant, qu'elle soit morale ou juridique.

À partir de ces éclairages théoriques et en lien avec la problématique, nous avons construit un dispositif de collecte déléments empiriques permettant d'observer la circulation des connaissances au sein de situations d'enseignement.

\section{Méthodologie}

\section{a) Présentation du dispositif de collecte d'éléments empiriques}

La supervision en direct relie des enseignants avec différents types de technologie pour intervenir en situation avec un feedback performant (Gallant et Thyer, 1989), notamment le "Bug in Ear» (BIE) qui est le dispositif le plus utilisé. Certains le nomment « oreillette sans fil » ou dispositif de troisième oreille mécanique, système électronique d'audio-repérage. Le BIE consiste en un procédé portable comprenant une radio oreillette et un microphone qui permet de coacher ou de superviser en temps réel pour donner un feedback immédiat. Nous avons conçu un dispositif de recueil d'éléments empiriques basé sur une forme de BIE que nous avons adapté aux besoins de notre étude. Ainsi, le dispositif de collecte d'éléments empiriques mis en place permet de donner des informations directement pendant la situation d'enseignement-apprentissage en classe, ce qui nous donne la possibilité d'observer la transformation de la connaissance chez les enseignants.

Ce dispositif se caractérise de la manière suivante (voir schéma 1). L'enseignant, face aux élèves, est équipé d'une oreillette sans fil en liaison avec un autre enseignant qui se situe dans une autre salle de l'établissement et qui observe la séance d'enseignement grâce à une retransmission (en temps réel) vidéo et audio. 


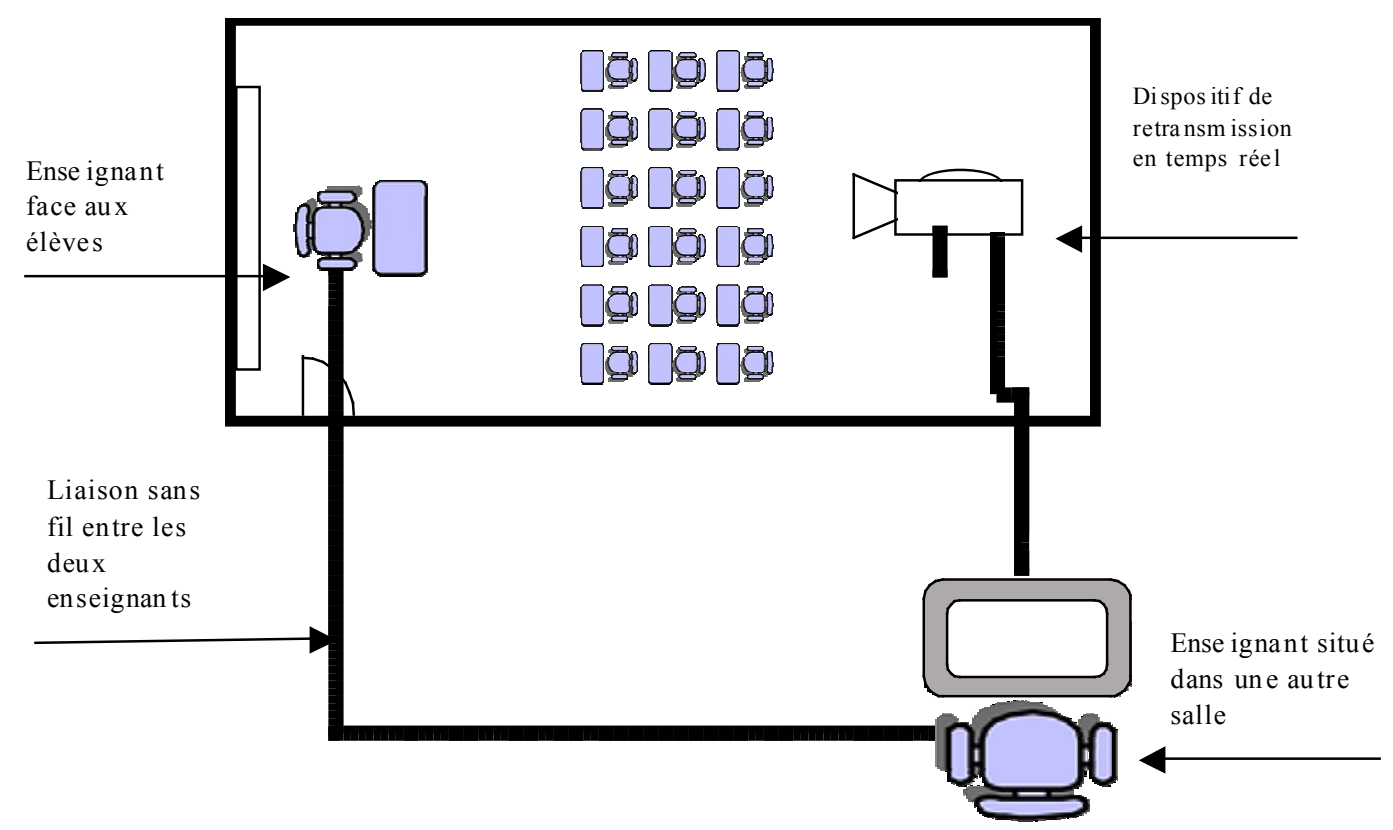

\section{Schéma 1}

Vue du dispositif de collecte d'éléments empiriques.

Le chercheur est extérieur au dispositif et en position d'observateur. Au-delà des discours relevés dans les entretiens et les grilles SEP comme il est précisé dans le paragraphe ci-dessous, les chercheurs ont observé les régulations faites entre enseignants sans intervenir. Dans les pratiques d'enseignement, la régulation s'apparente à la notion de guidance et d'accompagnement du geste professionnel. «Les mécanismes de régulation, de guidage, de contrôle, d'ajustement et de réorientation de l'action sont au centre de toutes les théories d'apprentissage »(Allal,2007). La régulation des pratiques d'enseignement en situation a pour objectif l'autonomisation progressive de l'enseignant novice. Cette régulation repose sur la transmission d'informations d'un enseignant en mobilisant ses savoirs vers un autre enseignant en situation de classe.

\section{b) Présentation de l'enquête}

Ce dispositif a été mis en place avec trois enseignants dans différentes situations d'enseignement comme la salle de classe, l'atelier pédagogique ou les parcelles agricoles (voir le schéma 2). Comme indiqué dans le schéma 1 , chacune de ces séances a été suivie en temps réel par un enseignant qui avait la possibilité de communiquer des informations directement dans la situation d'enseignementapprentissage. La collecte d'éléments empiriques s'est effectuée sur six séances, dans des établissements de l'enseignement agricole en France.

Avant chacune des séances d'enseignement, nous avons réalisé une évaluation du sentiment d'efficacité professionnelle des enseignants sur les différentes facettes du métier inhérentes à la situation d'enseignement qu'ils avaient prévu. Les données concernant le sentiment d'efficacité professionnelle 
ont été recueillies grâce à un questionnaire portant sur le sentiment de maittrise des différentes activités d'enseignement. Les différentes séances d'enseignement régulées par un autre enseignant ont été enregistrées ainsi que les informations communiquées par l'enseignant extérieur à la situation. À la suite de ces séances d'enseignement, les chercheurs ont procédé à un entretien semi-directif (à partir d'un guide) avec chacun des enseignants régulés portant sur le déroulement de la séance, leurs pratiques d'enseignement et chaque information reçue pendant la séance.

\begin{tabular}{|c|c|c|c|c|}
\hline Séances & Thèmes & Enseignants & Type de séance et lies & Classe \\
\hline 1 & Les semoirs en ligne & Jean & Cours & Baccalauréat Professionnel CGEA \\
\hline 2 & Les semoirs en ligne & Jean & Cours & Baccalunuéat Professionnel CGEA \\
\hline 3 & L'electricite embarqquete & Pierre & $\mathrm{TD}^{2}$ & $\begin{array}{l}\text { Baccalauréat Professionnel } \\
\text { Agrobquipements }\end{array}$ \\
\hline 4 & L'électricité embarquée & Pierre & TD & $\begin{array}{l}\text { Baccalauréat Professicenel } \\
\text { Agroéquipements }\end{array}$ \\
\hline 5 & $\begin{array}{l}\text { Matériels de travail du sol animé par } \\
\text { la prise de force }\end{array}$ & Bernard & $\mathrm{TP}^{\mathrm{s}}$ & BPREA $^{4}$ \\
\hline 6 & $\begin{array}{l}\text { Materiels de travail du sol animé par } \\
\text { la prise de force }\end{array}$ & Bernard & TP & BPREA \\
\hline
\end{tabular}

\section{Schéma 2}

Tableau des enseignants $N$ et situation d'enseignement de la collecte d'éléments empiriques

\section{c) Présentation des résultats}

Les résultats ont été regroupés sous la forme d'un tableau. La première colonne indique le sentiment d'efficacité professionnelle des enseignants à partir d'une échelle allant de 1 à 4 , le 1 représentant un sentiment de maîtrise plutôt faible et le 4 un sentiment de maîtrise plutôt fort. La deuxième colonne reprend les informations qu'ont reçues les enseignants liées aux différentes facettes du métier. Enfin, les discours sur les pratiques d'enseignement qui sont issus des entretiens post-séance sont également liés aux informations reçues et présentées en colonne 3. Dans le présent article, nous choisissions de présenter un extrait du tableau récapitulant les régulations, le SEP et les discours des enseignants sur leurs pratiques. Dans cet extrait, il s'agit de deux types d'informations reçues par les enseignants $\mathrm{N}$ au cours de leurs séances d'enseignement afin de montrer comment les éléments empiriques recueillis ont été regroupés. 


\section{Tableau 1}

Extraits des résultats, mise en relation du SEP, des régulations et des discours des enseignants.

\begin{tabular}{|c|c|c|c|c|c|}
\hline \multicolumn{4}{|c|}{$\begin{array}{l}\text { Sentiment đeficacité } \\
\text { professlonnelle }\end{array}$} & \multirow[b]{2}{*}{ Régulations } & \multirow[b]{2}{*}{ Extraits de vertatim issus de l'entretien post-stance } \\
\hline Items & 를 & $\frac{8}{8}$ & 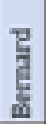 & & \\
\hline $\begin{array}{l}\text { L'adapeat } \\
\text { ion du } \\
\text { cours aux } \\
\text { niveau et } \\
\text { difficulté } \\
\text { s des } \\
\text { Etves }\end{array}$ & 4 & 4 & 3 & $\begin{array}{l}\text { - Ill faudrait que } \\
\text { tu montres oû } \\
\text { est-ce que ron } \\
\text { accroche le } \\
\text { semolr en travall } \\
\text { simplifie - }\end{array}$ & 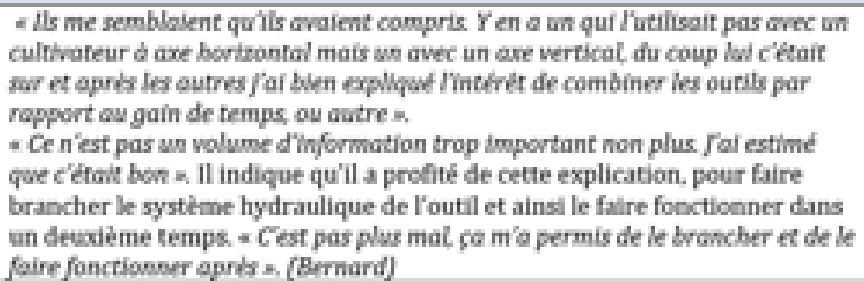 \\
\hline $\begin{array}{l}\text { L'utilisati } \\
\text { on de } \\
\text { supports } \\
\text { audiovis } \\
\text { uels, } \\
\text { informati } \\
\text { que }\end{array}$ & 3 & 4 & 4 & $\begin{array}{l}\text { - marque au } \\
\text { ubleau les mots } \\
\text { que tu fais écrire } \\
\text { aux élëves } \\
\text { - cannelures * et } \\
\text { - ergots * }\end{array}$ & 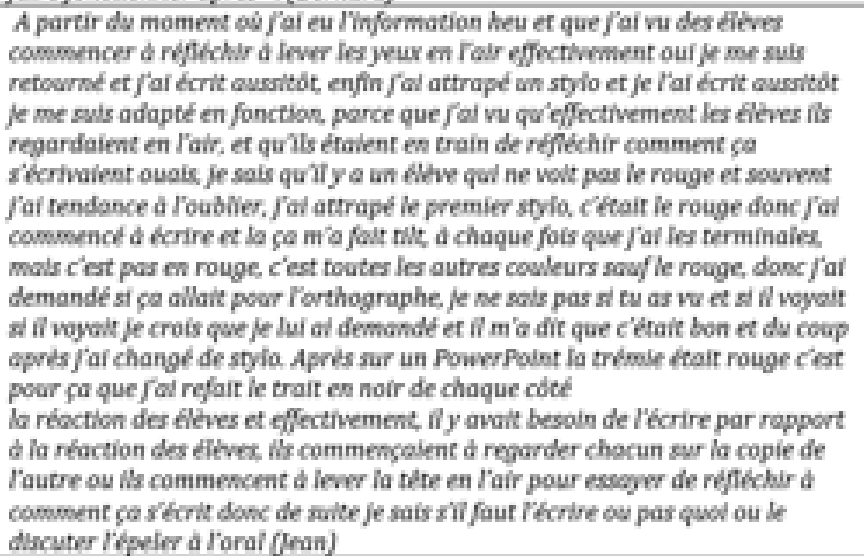 \\
\hline
\end{tabular}

L'analyse des résultats se base sur les liens entre le sentiment d'efficacité professionnelle des enseignants, le type de régulation reçu pendant les séances d'enseignement ainsi que les discours des enseignants post-séances sur les pratiques. Cette analyse qualitative des pratiques s'articule autour de la connaissance partagée et mobilisée et de l'action incarnée et éclairée par un sujet connaissant et agissant.

\section{Analyse et discussion : chemin de la connaissance interindividuelle et intra-individuelle}

À partir des modèles théoriques exposés dans la première partie, nous analysons les liens entre sentiment d'efficacité professionnelle, information reçue et réflexivité pour tenter de saisir le partage des connaissances dans l'action, et ce, en prenant en compte les dimensions du sujet, des savoirs et des pratiques. Dans un premier temps, nous présentons les verbatim issus des entretiens en relation avec les grilles SEP et les observations des régulations, catégorisés et classés selon les relations entre sujets et pratiques (plan onto-praxéologique), sujets et savoirs (plan onto-épistémologique) et enfin savoirs et pratiques (plan épistémo-praxéologique). 


\section{a) Conversion de la connaissance}

Le tableau ci-dessous résume la mise en relation de ces différents éléments. La première colonne explicite le type de régulations observées au cours des séances mises en relation avec le SEP des enseignants. Par rapport à cette interaction régulation-SEP, nous développons dans la deuxième colonne des éléments relatifs au plan onto-praxéologique, c'est-à-dire aux connaissances tacites qui ont émergé lors de cette régulation. La troisième colonne reprend le plan onto-épistémologique c'est-à-dire l'action modifiée et les connaissances en action. La dernière colonne concerne le plan épistémo-praxéologique, où au travers des discours des enseignants nous relevons le passage de l'action à la connaissance explicite.

\section{Tableau 2}

Conversion de la connaissance.

\begin{tabular}{|c|c|c|c|}
\hline Catégories de régulation et SEP & Axe 1 : plan onto-praxéologique & Axe 2 : plan onto-épistémologique & $\begin{array}{l}\text { Axe } 3 \text { : plan épistémo- } \\
\text { praxéologique }\end{array}$ \\
\hline \multirow[t]{2}{*}{$\begin{array}{l}\text { Didactique : contenu à expliciter } \\
\text { SEP : bonne maitrise adaptation du } \\
\text { cours au niveau des élèves }\end{array}$} & $\begin{array}{l}\text { " tu peux le faire tourner à la } \\
\text { main peut être? » « il faudrait } \\
\text { que tu montres où est-ce que } \\
\text { l'on accroche le semoir en travail } \\
\text { simplifié» } \\
\text { "Bernard, est-ce que tu as prévu } \\
\text { de montrer la cinématique de la } \\
\text { prise de force, je ne suis pas sûr } \\
\text { qu'elle ait compris la stagiaire, de } \\
\text { la prise de force jusqu'aux pièces } \\
\text { travaillantes» " est-ce que tu peux } \\
\text { expliquer davantage pourquoi ça } \\
\text { clignote vite quand il manque une } \\
\text { ampoule?» }\end{array}$ & $\begin{array}{l}\text { Action modifiée } \\
\text { J'ai quand même essayé de faire } \\
\text { tourner la transmission à la main } \\
\text { J'ai profité de cette explication, } \\
\text { pour faire brancher le système } \\
\text { hydraulique de l'outil } \\
\text { "J'ai repris organes par organes } \\
\text { pour qu'ils comprennent bien } \\
\text { l'intérêt de chaque organe, sortie du } \\
\text { tracteur donc prise de force, l'arbre } \\
\text { de transmission à cardans,... ". }\end{array}$ & $\begin{array}{l}\text { "Je me suis rendu compte qu'une } \\
\text { élève n'avait pas compris le } \\
\text { fonctionnement des différents } \\
\text { montages de dents } \\
\text { L'enseignant indique quill n'avait } \\
\text { pas prévus. J'ai ensuite donné } \\
\text { une explication plus scientifique, } \\
\text { plus technique, différente et plus } \\
\text { cohérente. Je pensais que la réponse } \\
\text { quej'avais donnée a l'élève était } \\
\text { suffisante même sij'avais senti } \\
\text { qu'elle interrogeait les élèves car ils } \\
\text { s'agitaient. » }\end{array}$ \\
\hline & $\begin{array}{l}\text { Précision sur le savoir en fonction } \\
\text { des élèves } \\
\text { Connaissance sur l'anticipation } \\
\text { des difficultés des élèves par } \\
\text { rapport au savoir enseigné }\end{array}$ & $\begin{array}{l}\text { Mobilisation de la connaissance } \\
\text { tacite émergente dans l'action } \\
\text { entraînant sa modification } \\
\text { Adaptation du savoir enseigné au } \\
\text { niveau de difficultés des élèves } \\
\text { en proposant une autre stratégie } \\
\text { didactique }\end{array}$ & $\begin{array}{l}\text { Action non prévue dans la } \\
\text { préparation } \\
\text { Conscientisation de l'action et } \\
\text { réflexivité } \\
\text { Anticiper le niveau de difficultés } \\
\text { des élèves pour adapter l'ingénierie } \\
\text { didactique } \\
\text { Connaissance sur les difficultés des } \\
\text { élèves et sur les modes d'adaptation } \\
\text { didactique }\end{array}$ \\
\hline
\end{tabular}




\begin{tabular}{|c|c|c|c|}
\hline \multirow[b]{2}{*}{$\begin{array}{l}\text { Pédagogique : gestion de l'indiscipline } \\
\text { en classe } \\
\text { SEP : très bonne maitrise }\end{array}$} & $\begin{array}{l}\text { "Pierre à ta gauche au fond je } \\
\text { ne suis pas sûr qu'il suit l'élève } \\
\text { en vert» } \\
\text { "Pierre je crois que ce serait bien } \\
\text { que tu vois ceux de derrière, je crois } \\
\text { qu'ils font un peu les idiots» }\end{array}$ & $\begin{array}{l}\text { "J'ai observé l'élève afin de voir s'il } \\
\text { notait ou pas. » } \\
\text { "Quand je les ai observés, J'ai } \\
\text { perçu qu'ils étaient agités, qu'ils } \\
\text { discutaient entre eux.» }\end{array}$ & $\begin{array}{l}\text { "Je me suis rendu compte que l'elève } \\
\text { avait la tête levée. Pour moi, ily } \\
\text { avait donc une éventualité pour que } \\
\text { cet élève soit en retard sur la prise } \\
\text { de notes. J'ai donc décidé, de me } \\
\text { rapprocher de l'élève. } \\
\text { J'ai donc décidé d'agir afin de } \\
\text { stopper l'agitation. Pour cela, je les ai } \\
\text { regardés, je leur ai souri et je suis allé } \\
\text { m'installer à côté d'eux. " }\end{array}$ \\
\hline & $\begin{array}{l}\text { Identification de comportement } \\
\text { non propice aux apprentissages } \\
\text { Connaissance relative aux } \\
\text { comportements des élèves par } \\
\text { rapport à l'apprentissage }\end{array}$ & $\begin{array}{l}\text { Action modifiée grâce à } \\
\text { l'information transmise } \\
\text { Identification de comportements } \\
\text { non propices aux apprentissages } \\
\text { et adaptation de la pratique } \\
\text { pédagogique }\end{array}$ & $\begin{array}{l}\text { Action prévue mais non réalisée } \\
\text { dans la situation } \\
\text { Conscientisation de l'action, } \\
\text { réflexivité } \\
\text { Connaissance sur le repérage } \\
\text { des comportements propices à } \\
\text { l'apprentissage } \\
\text { Connaissance sur les } \\
\text { comportements des élèves en } \\
\text { rapport avec l'apprentissage } \\
\text { et sur les modes d'adaptation } \\
\text { pédagogique liés à la gestion de } \\
\text { la classe }\end{array}$ \\
\hline \multirow[t]{2}{*}{$\begin{array}{l}\text { Pédagogique : utilisation des supports } \\
\text { SEP : bonne maitrise }\end{array}$} & $\begin{array}{l}\text { "marque au tableau les mots } \\
\text { que tu fais écrire aux élèves } \\
\text { "cannelures » et « ergots» Jean }\end{array}$ & $\begin{array}{l}\text { "J'ai pris des informations dans } \\
\text { la classe, j'ai vu que les élèves } \\
\text { avaient la tête levée, pour moi cela } \\
\text { signifie que les élèves étaient en } \\
\text { train de réfléchir à l'orthographe } \\
\text { des mots. J'ai vu qu'effectivement } \\
\text { les élèves, ils regardaient en l'air, et } \\
\text { qu'ils étaient en train de réfléchir } \\
\text { comment ça s'écrivaient. } \\
\text { J'ai décidéde prendre un stylo afin } \\
\text { de noter les mots au tableau. Ce n'est } \\
\text { pas dans ma pratique habituelle de } \\
\text { faire cela, j'ai plutôt l'habitude de } \\
\text { dicter les mots et ensuite d'épeler à } \\
\text { l'oral ceux qui paraissent difficiles à } \\
\text { écrire pour les élèves.» }\end{array}$ & $\begin{array}{l}\text { «les élèves finissent le cours avec } \\
\text { un circuit qui n'est pas complet mais } \\
\text { qui est fonctionnel et c'est pour moi } \\
\text { l'essentiel. Cela aura un impact sur } \\
\text { le cours suivant puisque je devrai } \\
\text { terminer le circuit complètement avec } \\
\text { les élèves» }\end{array}$ \\
\hline & $\begin{array}{l}\text { Variation de l'activité des élèves } \\
\text { Mobilisation des élèves dans les } \\
\text { apprentissages } \\
\text { Connaissance relative aux } \\
\text { différents modes d'apprentissage } \\
\text { des élèves }\end{array}$ & $\begin{array}{l}\text { Identification et utilisation de } \\
\text { supports favorisant l'apprentissage } \\
\text { des élèves }\end{array}$ & $\begin{array}{l}\text { Connaissance des supports } \\
\text { propices à l'apprentissage des } \\
\text { élèves sur les modes d'adaptation } \\
\text { pédagogiques de l'enseignant }\end{array}$ \\
\hline
\end{tabular}




\begin{tabular}{|c|c|c|c|}
\hline \multirow[t]{2}{*}{$\begin{array}{l}\text { Pédagogique : suivi des apprentissages } \\
\text { des élèves } \\
\text { SEP : très bonne maitrise }\end{array}$} & $\begin{array}{l}\text { "prends un élève pour expliquerce } \\
\text { qu'il a retenu " } \\
\text { "Pierre cible les questions, pour } \\
\text { que ce ne soit pas toujours les } \\
\text { mêmes qui répondent" }\end{array}$ & 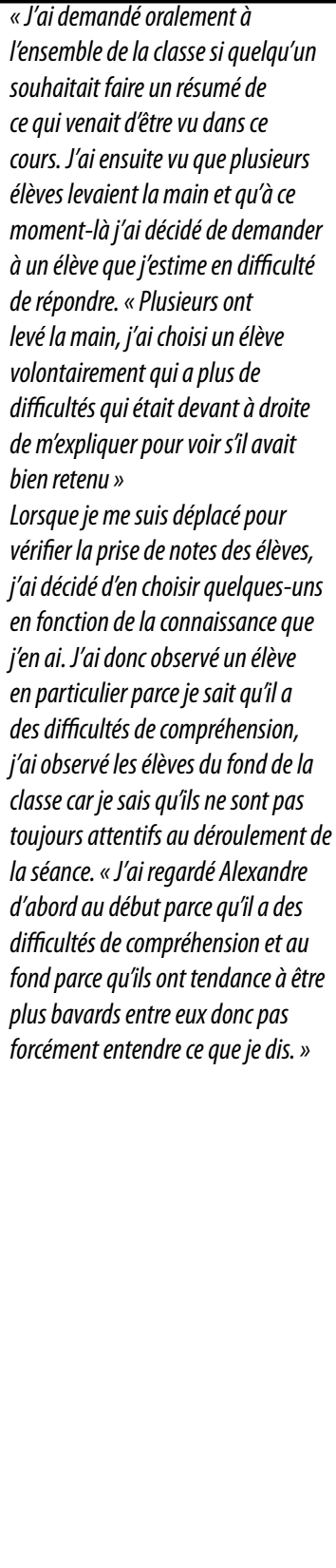 & $\begin{array}{l}\text { L'enseignant indique que dans } \\
\text { l'avenir, il gardera cinq minutes } \\
\text { à la fin de la séance pour faire } \\
\text { participer les élèves pour voir ce } \\
\text { quíls ont retenu de la séance. «ll } \\
\text { faudra prévoir un peu plus de temps } \\
\text { au moins cinq minutes pour faire } \\
\text { participer les élèves pour voir ce qu'ils } \\
\text { ont retenu à la fin ». } \\
\text { II n'avait pas prévu d'interroger un } \\
\text { élève en particulier. II l'a fait, car, } \\
\text { explique-t-ill, il a l'habitude dele } \\
\text { faire. En règle générale, il dit poser } \\
\text { des questions aux élèves afin de } \\
\text { faire ressortir leurs connaissances } \\
\text { et ensuite il les organise. "J'ai } \\
\text { l'habitude toujours de me servir des } \\
\text { connaissances des élèves, donc je suis } \\
\text { un peu le chef d'orchestre, j'organise } \\
\text { leurs connaissances». } \\
\text { L'enseignant précise qu'il a vu que } \\
\text { l'élève n'écoutait pas forcément. } \\
\text { En lui posant une question, il lui } \\
\text { signifiait qu'ill'avait vu bavarder et } \\
\text { qu'en l'interpellant il lui demandait } \\
\text { de rendre des comptes en répondant } \\
\text { à la question. Il rajoute qu'il a guidé } \\
\text { l'élève parce que celui-ci ne savait pas } \\
\text { trop, il a donc reposéles choses en } \\
\text { partant de la photo de l'interrupteur } \\
\text { à bascule mais il dit que l'élève a } \\
\text { répondu sur le bouton poussoir qui } \\
\text { était la réponse à la deuxième photo. } \\
\text { L'enseignant dit alors qu'il décide } \\
\text { d'alller dans son sens, c'est à dire de } \\
\text { commencer par le bouton poussoir } \\
\text { et ensuite de revenir à l'interrupteur } \\
\text { à bascule. L'enseignant explique qu'il } \\
\text { est allé dans son sens car la réponse } \\
\text { de l'élève n'était pas forcément } \\
\text { fausse, même si ce n'était pas celle } \\
\text { qu'il attendait puisque cétait la } \\
\text { suivante. L'enseignant dit en avoir } \\
\text { profité pour la traiter en premier. }\end{array}$ \\
\hline & $\begin{array}{l}\text { Techniques d'évaluation des } \\
\text { apprentissages } \\
\text { Connaissance relative à } \\
\text { l'évaluation des apprentissages } \\
\text { des élèves pendant la séance }\end{array}$ & $\begin{array}{l}\text { Identification des différents modes } \\
\text { d'évaluation des apprentissages } \\
\text { des élèves pendant la séance }\end{array}$ & $\begin{array}{l}\text { Connaissance sur l'évaluation } \\
\text { personnalisée dans le suivi des } \\
\text { apprentissages et sur les modes } \\
\text { d'adaptation pédagogique liés aux } \\
\text { suivis des apprentissages des élèves }\end{array}$ \\
\hline
\end{tabular}


Les régulations en situations ont été regroupées en deux catégories, les aspects pédagogiques et les aspects didactiques. Les aspects pédagogiques sont relatifs à la relation à l'élève, à l'organisation de la séance et au matériel tandis que les aspects didactiques sont relatifs aux savoirs. Cette dichotomie n'est utile qu'à l'analyse, mais ne renvoie pas à une volonté théorique qui reflèterait une posture épistémologique, car le savoir ne peut être partagé que dans une interaction. La pédagogie s'intéresse également aux savoirs et la didactique n'exclut pas les élèves et les enseignants de ses analyses. Cependant, cette catégorisation renvoie à la définition des savoirs professionnels telle qu'elle est exposée dans la partie théorique, c'està-dire une typologie de construits par les praticiens à partir des informations échangées.

\section{b) Le sentiment d'efficacité professionnelle}

Le SEP donne un indicateur de persévérance et d'implication dans la tâche. Lorsque le SEP est élevé, l'enseignant semble accepter les propositions de changement de sa pratique, en modifiant immédiatement son action. On pourrait donc dire que c'est le niveau de croyance dans la possible réalisation de la tâche qui permet de modifier l'action et qui autorise un processus de réflexivité.

Autrement dit, la conversion de la connaissance dans l'action se révèle possible d'autant plus que le SEP est élevé permettant ainsi à la connaissance tacite d'être convertie en connaissance explicite grâce au double processus de modification de l'action et de l'engagement dans la réflexion qui fait écho à un certain degré d'engagement dans la tâche.

On voit donc ici que les interactions entre sujet, savoirs et pratiques concrétisées dans les différents plans, le plan onto-praxéologique, le plan onto-épistémologique et le plan épistémo-praxéologique, permettent d'appréhender la part interindividuelle et intra-individuelle dans les liens entre la pratique et la connaissance.

Si nous reprenons les éléments rassemblés dans le tableau ci-dessus, nous pouvons tenter de cerner ce qui est en jeu au travers de chacun des axes de la conversion de la connaissance dans l'action tels que nous les avons déterminés.

\section{c) Axe 1:plan onto-praxéologique}

Du point de vue didactique, que nous pouvons qualifier dans les régulations par les contenus nécessitant plus d'explicitation, nous voyons que certaines connaissances tacites de l'enseignant $\mathrm{E}$ ont émergé au travers de suggestions concernant le lien entre le savoir enseigné et son appropriation par les élèves. Alors que l'enseignant $\mathrm{N}$ en situation de classe avait déclaré avoir une bonne maîtrise de l'adaptation du cours au niveau des élèves, l'anticipation des difficultés perçues par rapport au savoir enseigné semble résulter ici d'une connaissance tacite non partagée.

Du point de vue pédagogique, concrétisé dans les régulations par la gestion de l'indiscipline en classe, l'enseignant $\mathrm{E}$ fait émerger, au travers de l'identification de comportements non propices aux apprentissages, une connaissance tacite relative aux comportements des élèves dans le processus d'apprentissage. L'enseignant $\mathrm{N}$, bien que déclarant avoir une très bonne maîtrise de ces aspects de gestion de l'indiscipline en classe, ne perçoit pas à ce moment-là la nécessité de les identifier pour adapter sa pratique. 
Toujours du point de vue pédagogique dans les régulations, mais concernant l'utilisation de supports, l'enseignant E propose de vérifier l'activité des élèves pour mieux les mobiliser dans l'apprentissage, révélant ainsi une connaissance tacite relative aux différents modes d'apprentissage des élèves.

Enfin, concernant le suivi des apprentissages des élèves, l'enseignant $\mathrm{N}$ déclare en avoir une très bonne maîtrise; pourtant, l'enseignant $\mathrm{E}$ introduit des techniques d'évaluation des apprentissages qui montrent une connaissance tacite relative à l'évaluation des apprentissages des élèves pendant la séance non partagée avant cette régulation.

\section{d) Axe 2:plan onto-épistémologique}

$\mathrm{Du}$ point de vue didactique, l'enseignant $\mathrm{N}$ en situation semble mobiliser la connaissance tacite émergente au travers de la modification de son action. Son sentiment de bonne maîtrise de cet aspect lui permet ici d'adapter le savoir enseigné au niveau de difficulté des élèves en proposant une autre stratégie didactique.

Concernant la gestion de l'indiscipline dans la classe, à travers l'identification de comportements non propices aux apprentissages, l'enseignant $\mathrm{N}$ en situation modifie et adapte sa pratique pédagogique. De même, en identifiant la nécessité de varier les supports, il introduit une modification des modalités d'enseignement pour favoriser l'apprentissage des élèves.

De manière très proche, par la perception des différents modes d'évaluation des apprentissages, il diversifie son approche par des évaluations successives du niveau de compréhension des élèves, son très bon sentiment de maîtrise du suivi des apprentissages des élèves amène un engagement propice à cette adaptation.

\section{e) Axe 3 :plan épistémo-praxéologique}

La conscientisation émergente de l'action modifiée nous paraît être un signe d'un processus naissant de réflexivité qui permet d'anticiper le niveau de difficulté des élèves pour adapter l'ingénierie didactique. Autrement dit, l'enseignant $\mathrm{N}$ en situation semble développer une connaissance explicite sur les difficultés des élèves et sur les modes d'adaptation didactique.

$\mathrm{Du}$ point de vue pédagogique, l'enseignant développe une connaissance explicite sur le repérage des comportements propices à l'apprentissage. Il s'appuie sur son sentiment de très bonne maîtrise pour mieux prendre en compte les comportements d'indiscipline et trouver des modes d'adaptation pédagogique liés à la gestion de la classe.

La connaissance des supports propices à l'apprentissage des élèves, en se diversifiant, provoque un changement de pratiques projeté également sur les séances d'enseignement suivantes. Le sentiment de très bonne maîtrise du suivi des apprentissages des élèves, en se heurtant à la prise de conscience de la nécessaire personnalisation dans le suivi des apprentissages, amène une connaissance plus explicite sur l'évaluation des apprentissages et modifie les modes pédagogiques liés aux suivis des élèves. 
L'analyse des résultats proposée ici montre les interrelations constructives entre sujets et savoirs, sujet et pratiques, savoirs et pratiques à partir d'éléments théoriques reposant sur les processeurs des pratiques d'enseignement, les savoirs professionnels et la dynamique de la transformation de la connaissance organisationnelle au travers d'un dispositif de régulation en situation.

\section{Conclusion}

La question de la formation des enseignants novices en situation se pose de manière de plus en plus cruciale au vu des réformes récentes qui instaurent une entrée rapide dans le métier. La fonction d'accompagnement se voit ainsi réinterrogée notamment en regard de la construction de savoirs professionnels. Un dispositif de régulation en situation entre enseignant expérimenté et enseignant novice permet de voir, en première approche, comment la connaissance en circulation dans ce type de dispositif se transforme et transforme les pratiques d'enseignement, et ce, en relation avec le sentiment d'efficacité professionnelle. Nous avons montré que, dans les différentes catégories de régulation (didactique, pédagogique), nous pouvions mettre au jour une transformation de la connaissance sur le plan onto-praxéologique, le plan onto-épistémologique et enfin le plan épistémo-praxéologique. L'étude de ces différents axes montre à partir de la modification de l'action l'émergence de connaissances tacites qui, en s'explicitant, se transforment (sont partagées) et permettent une appropriation qui ouvre, à partir d'un processus réflexif, vers la construction de nouvelles connaissances.

\section{Références}

Allal, L. (2007). Introduction. Régulations des apprentissages : orientations conceptuelles pour la recherche et la pratique en éducation. Dans L. Allal et L. Mottier Lopez (dir.), Régulation des apprentissages en situation scolaire et en formation (p. 7-23). Bruxelles : De Boeck Supérieur. http://dx.doi.org/10.3917/dbu.motti.2007.01.0007

Astolfi, J.-P. (1995). Vers une pédagogie constructiviste. Lyon : Association Voies Livres.

Bandura, A. (1977). Self-efficacy: Toward a unifying theory of behavioral change. Psychological Review, 84(2), 191-215. http://dx.doi.org/10.1037/0033-295x.84.2.191

Bandura, A. (1986). Social foundations of thought and action: a social cognitive theory. Englewood Cliffs, NJ : Prentice Hall.

Bandura, A. (2003). Auto-efficacité : le sentiment d'efficacité personnelle. Bruxelles : De Boeck.

Bru, M. (2004). Les pratiques enseignantes comme objet de recherche. Dans J.-F. Marcel et P. Rayou (dir.), Recherches contextualisées en éducation (p. 281-299). Paris : Institut national de la recherche pédagogique.

Carré, P. (2004). Bandura : une psychologie pour le XXI siècle?. Revue Savoirs, (Hors série), 9-50. http://dx.doi. org/10.3917/savo.hs01.0009

Fauré, L. (2014). Régulation du pouvoir d'agir au sein des pratiques d'enseignement des enseignants en sciences et techniques des agroéquipements (Mémoire de master, Université de Toulouse). Repéré à http://sites.ensfea.fr/cdi/wp-content/ uploads/sites/3/2014/07/M\%C3\%A9moire-Version-VF1.pdf

Foucault, M. (1975). L’archéologie du savoir. Paris : Gallimard.

Gallant, J. P. et Thyer, B. A. (1989). The "bug-in-the-ear" in clinical supervision: A review. The Clinical Supervisor, 7(2/3), 43-58. http://dx.doi.org/10.1300/j001v07n02 04

Gardiès, C. (2012). Dispositifs info-communicationnels de médiation des savoirs : cadre d'analyse pour l'informationdocumentation. Repéré à http://oatao.univ-toulouse.fr/9862/1/Gardies_9862.pdf 
Gardiès, C. et Marcel, J.-F. (2013). On the praxeological dimension of organizational knowledge conversion: The example of a "professionalisation year" for trainee teachers in French agricultural education. International Journal of Information Technology and Management, 12(3/4), 226-251. http://dx.doi.org/10.1504/ijitm.2013.054797

Gauthier, C., Desbiens, J.-F., Malo, A., Martineau, S. et Simard, D. (1997). Pour une théorie de la pédagogie : recherches contemporaines sur le savoir des enseignants. Sainte-Foy, QC : Presses de l'Université Laval.

Gillet, G. et Fauré, L. (2014). Sciences et techniques des agroéquipements : quelle didactique pour quelle discipline?. Dans C. Gardiès, et N. Hervé (dir.), L'enseignement agricole entre savoirs professionnels et savoirs scolaires. Les disciplines en question (p. 23-42). Dijon : Éducagri Éditions.

Jeanneret, Y. (2000). Ya-t-il (vraiment) des technologies de l'information?. Villeneuve-d'Ascq : Presses universitaires du Septentrion.

Lefeuvre, G. (2005). L'accès aux pratiques d'enseignement à partir d'une double lecture de l'action. Journal international sur les représentations sociales, 2(1), 78-88. Repéré à http://geirso.uqam.ca/jirso/Vol2 Aout05/78Lefeuvre.pdf

Marcel, J.-F. (2002). La connaissance de l'action et des pratiques enseignantes. Dans J.-F. Marcel (dir.), Les sciences de l'éducation, des recherches, une discipline (p. 79-112). Paris : L'Harmattan.

Marcel, J.-F. (2004). Les pratiques enseignantes hors de la classe. Paris : L'Harmattan.

Marcel,J.-F. (2005). Apprendre en travaillant. Contribution à une approche socio-cognitive du développement professionnel de l'enseignant. Université Toulouse - le Mirail.

Marcel, J.-F. (2009). Le sentiment d'efficacité professionnelle, un indicateur pour connaître le développement professionnel des « nouveaux » professeurs de l'enseignement agricole français. Questions vives, 5(11), 161-176. http://dx.doi. org/10.4000/questionsvives. 564

Marcel,J.-F. (2014). Les pratiques enseignantes mises en récit. Recherches en éducation, (19), 82-95. Repéré à http://www. recherches-en-education.net/IMG/pdf/REE-no19.pdf

Marcel, J.-F. et Gardiès, C. (2010). La difficile construction de l'identité professionnelle des professeurs-documentalistes de l'enseignement agricole public. Recherches en éducation, (10), 146-160. Repéré https://hal.archives-ouvertes.fr/hal$\underline{01280288 / \text { document }}$

Nonaka, I. (1994). A dynamic theory of organizational knowledge creation. Organization Science, 5(1), 14-37.

Nonaka, I. et Peltokorpi, V. (2006). Objectivity and subjectivity in knowledge management: A review of 20 top articles. Knowledge and Process Management, 13(2), 73-82. http://dx.doi.org/10.1002/kpm.251

Nonaka, I. et Von Krogh, G. (2009). Perspective - tacit knowledge and knowledge conversion: Controversy and advancement in organizational knowledge creation theory. Organization Science, 20(3), 635-652. http://dx.doi. org/10.1287/orsc. 1080.0412

Perrenoud, P. (2000). D'une métaphore à l'autre : transférer ou mobiliser ses connaissances?. Dans J. Dolz et E. Ollagnier, (dir.), Lénigme de la compétence en éducation (p. 45-60). Bruxelles : De Boeck.

Rock, M. L., Gregg, M., Thead, B. K., Acker, S. E., Gable, R. A. et Zigmond, N. P. (2009). Can you hear me now? Evaluation of an online wireless technology to provide real-time feedback to special education teachers-in-training. Teacher Education and Special Education, 32(1), 64-82. http://dx.doi.org/10.1177/0888406408330872

Safourcade, S. (2010). Du sentiment d'efficacité personnelle aux actes professionnels. Recherche et formation, (64), 141-156. http://dx.doi.org/10.4000/rechercheformation. 230

Tardif, M. et Gauthier, C. (1996). L'enseignant comme « acteur rationnel » : quelle rationalité, quel savoir, quel jugement?. Dans L. Paquay et al. (dir.), Former des enseignants professionnels. Quelles stratégies? Quelles compétences? (p. 209-237). Bruxelles : De Boeck.

Tardif, M. et Lessard, C. (1999). Le travail enseignant au quotidien : expérience, interactions humaines et dilemmes professionnels. Bruxelles : De Boeck Université. 
Tochon, F. V. (2004). La construction de l'expert en éducation : vers de nouvelles définitions. Recherche et formation, 47(1), 5-8. Repéré à http://www.persee.fr/doc/refor 0988-1824 2004 num 4711923

Wanlin, P. et Crahay, M. (2012). La pensée des enseignants pendant l'interaction en classe. Une revue de la littérature anglophone. Éducation et didactique, 6(1), 9-46. Repéré à https://educationdidactique.revues.org/1287

\section{Notes}

1 Master MEEF : Master Métier de l'enseignement, de l'éducation et de la formation.

2 Enseignants en STAE : Les enseignants en sciences et techniques des agroéquipements sont des enseignants de l'enseignement agricole français. Cette discipline concourt à former les élèves à l'utilisation et à la maintenance des équipements des exploitations agricoles.

3 Les conseillers pédagogiques : ils sont des enseignants expérimentés en activité dans les établissements de l'enseignement agricole et ils participent à la formation en situation des enseignants novices.

4 Réforme de la masterisation : En France, depuis 2014, les enseignants entrants doivent être titulaires d'un Master MEEF (Métier de l'enseignement, de l'éducation et de la formation). La deuxième année de ce diplôme se déroule pendant l'année de formation des enseignants.

\section{Pour citer cet article}

Faure, L., Gardiès, C., Marcel, J-F. (2017). Formation des enseignants : apprentissages professionnels d'enseignants novices au travers de régulations en situation de classe. Formation et profession, 25(3), 72-89.

http://dx.doi.org/10.18162/fp.2017.403 